\title{
Current Situation of TCM Treatment of Schizophrenia
}

\author{
Chen Lin*
}

Wenzhou Medical University, Wenzhou 325035, China. E-mail: 937391056@qq.com

Abstract: Schizophrenia is a common clinical disease in the department of psychiatry. Traditional Chinese medicine treatment of schizophrenia has a long history, rich experience, small side effects, to a certain extent can alleviate the condition of patients with schizophrenia. This article reviews the current situation of TCM treatment of schizophrenia in recent years from three aspects: TCM treatment, acupuncture treatment and catgut embedding treatment.

Keywords: Schizophrenia; TCM Treatment

Schizophrenia is a common cause of unknown serious mental illness, with greater social harm. There is no "schizophrenia" in the ancient Chinese medicine literature, according to its clinical manifestations, it is classified as "epilepsy" category. With the rapid development of society and the continuous increase of people's mental pressure, the incidence of schizophrenia is on the rise ${ }^{[1]}$. Due to the high safety of TCM treatment, the majority of patients will accept and choose this method to treat schizophrenia.

\section{Understanding of schizophrenia in Chinese medicine}

Both epilepsy and mania are clinical manifestations of mental disorders. The textbook of the tenth edition of Internal Medicine of Traditional Chinese Medicine (edited by Boli Zhang and Mianhua Wu) has a clear definition: epilepsy is characterized by mental depression, indifferent expression, silence, incoherent speech, quietness and inactivity. Crazy syndrome is characterized by mental excitement, manic rigid violence, disturbance and restlessness, destruction, beating and swearing, and more anger. The two clinical symptoms coexisting cannot be completely separated, so they are called madness. The earliest written records of diseases related to madness can be found in the Book of History, Weizi, written in the late Yin Dynasty (about the 11th century BC). The first name of madness is found in the Huangdi Neijing, which has a systematic description of its symptoms, etiology, pathogenesis and treatment. For example, in "Su Wen - Pulse to Subtle", clothes are not collected, words good and evil, do not avoid friends and relatives, this god's chaos. Describes the symptoms of the patient at the time of onset; in terms of the pathogenesis of madness, "all manias belong to fire" in the book "Su Wen - On the Importance of Truth" describes that the evil fire and the heart can cause the disease. "Yang Qi is on the top, Yin Qi is on the bottom, the bottom is empty and the top is solid, so it is called crazy disease" in Su Wen·Mai Jie, which describes that the imbalance of Yin and Yang can cause disease. "Su Wen·Tiao Jing Lun" in "more blood, then anger, lack of laughter", describes the disorder of qi and blood can cause disease. In "The Madness of

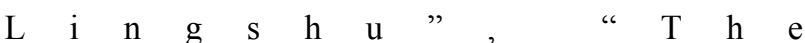

Copyright (C) 2020 Chen Lin

doi: 10.18686/aem.v9i4.177

This is an open-access article distributed under the terms of the Creative Commons Attribution Non-Commercial License (http://creativecommons.org/licenses/by-nc/4.0/), which permits unrestricted non-commercial use, distribution, and reproduction in any medium, provided the original work is properly cited. 
of Hunger", "The Great Fear of Gain”, "The Great Joy of Gain", and in "Su Wen - Strange Disease", "People born with a disease, what is the name of the disease, and what is it ?" Qi Bo said, "The disease is called fetal disease. When the disease is acquired in the womb, the mother is somewhat shocked." In Su Wen·Xuanming's Five Qi Theory, "The five evils are disordered; when the evils enter the Yang, they are mad; when they beat the Yang, they are epileptic.", "Su Wen • On the Alternation of Qi", "The fire is too hot in the New Year, the heat is popular illness and delirium." and so on respectively from the sentiment, the innate heredity, the exogenous pathogenic factors, the seasonal and so on discussed the madness disease etiology. "Su Wen •Disease can discuss" in "How cure ? Qi Bo said: take actually already make it drink pig iron." and so on discussed the treatment of madness ${ }^{[2]}$.

\section{Traditional Chinese medicine treatment}

Traditional Chinese medicine has certain effect in the treatment of schizophrenia. Chenxia Liu, et al. ${ }^{[3]}$ selected 60 patients with chronic schizophrenia and randomly divided them into 2 groups with 30 patients in each group. Patients in the treatment group alone were treated with aripiprazole; the combined treatment group was given traditional Chinese medicine decoction on the basis of the single treatment group. Specific prescription of TCM decoction: Poria cocos 15g, Pachyrhizus 12g, Radix Bupleuri 10g, Rhizoma Chuanxiong 10g, Pinellia tuber $10 \mathrm{~g}$, rehmannia root $10 \mathrm{~g}$, jujube seed $10 \mathrm{~g}$, Yuanzhi 10g, Xiangfu 10g, peach kernel 10g, Dannanxing $10 \mathrm{~g}$, tangerine peel $10 \mathrm{~g}$, Paeonia lactiflora $10 \mathrm{~g}$, liquorice $10 \mathrm{~g}$. Decocted in water for oral dose, 1 dose/d, 2 times/d, 4 weeks for a course of treatment, a total of 3 courses of treatment. After 6 and 12 weeks of treatment, SQLS score of patients in the group of TCM decoction combined with aripiprazole was significantly lower than the group of aripiprazole alone, indicating that TCM decoction combined with aripiprazole could significantly improve patients' sense of self-efficacy and improve the quality of their life. Shuhua $\mathrm{Wu}^{[4]}$ randomly divided 60 patients into the control group and the observation group with 30 patients each. Sulpiriol 200mg was taken orally twice a day in both groups. Eight weeks is a course of treatment. The observation group was treated with tradi- tional Chinese medicine. Take 1 dose daily, decocted in water for 3 times, for a course of treatment for 15 days, take 2 courses continuously. The total effective rate of the observation group was $96.7 \%$. The total effective rate of the control group was $80.0 \%$. The difference of total effective rate between the two groups was statistically significant $(\mathrm{P}<0.05)$. The results showed that the combined treatment of traditional Chinese and western medicine plays a role in mending depression, not only improves the clinical cure rate, but also reduces the adverse drug reactions. Jinming Huang ${ }^{[5]}$ self-designed clam the nerves and control epilepsy soup (American ginseng, Fushen, fried jujube seed, white peony root, dens draconis, pig iron fall, etc.) has the effect of soothing the nerves and nourishing brain, dispersing liver and relieving depression, and dredging collaterals and relieving phlegm. He thinks the syndrome of asthenia in origin and asthenia in superficiality and the treatment of symptoms and causes should be taken into comprehensive consideration. After 3 courses of treatment, 12 of the 32 patients were cured, with a total effective rate of $93.75 \%$ and a cure rate of $37.5 \%$, and no side effects, which were easily accepted by the patients.

\section{Acupuncture treatment}

\subsection{Acupuncture treatment}

Guimei Zhao ${ }^{[6]}$ reported the clinical effect of acupuncture combined with low-dose antipsychotics in the treatment of schizophrenia. A total of 50 patients were compared before and after treatment. Acupuncture at these points: Baihui, Shenting, Shangxing, Fenglong, Taichong, Shenmen, Sanyinjiao, Shanzhong, GuanYuan, in addition, connected to the electroacupuncture instrument, 30 mins each treatment, 3 times a week, the treatment course is 4 months. During the treatment with conventional low-dose clozapine. After treatment, the total score of positive and negative symptom scale (PANSS), negative scale and general psychopathology scale and Hamilton anxiety scale (HAMA) were significantly reduced, and the differences were significant at different time points $(\mathrm{P}<0.05)$. It shows that it can improve mental symptoms and relieve anxiety. Wei Liang ${ }^{[7]}$ reported that acupuncture has a good effect on patients with chronic schizophrenia. A total of 58 patients were selected and randomly divided into the control group and 
the observation group. The control group received oral olanzapine alone, while the observation group received combined acupuncture treatment (Taichong, Renzhong, Dazhui, Daling, Quchi, Fengfu, Neiguan and Fenglong). Acupuncture treatment was performed once a day for $30 \mathrm{~min}$ each time and the course of treatment was 8 weeks. After treatment, two groups of patients of SOD (superoxide dismutase) and MDA (malondialdehyde) levels drop, GPX (glutathione peroxidase) activity decreased, alzheimer's disease pathological behavior evaluation form (BEHAVE-AD) scores are to a certain extent reduce the immune indexes such as $\mathrm{CD} 3+, \mathrm{CD} 4+, \mathrm{CD} 8$ ,$+ \mathrm{IgG}$ and so on which levels are rising to a certain extent. The indicators in the observation group of patients with optimal range are more obvious. There were significant differences between the two groups $(\mathrm{P}<0.05)$. It shows that acupuncture can improve oxygen free radical metabolism, harsh immune resistance, and thus improve the symptoms of patients. Jihong $\mathrm{Wu}^{[8]}$ believed that through dialectical application of needles and combined use of various points, the effect of strengthening the spleen, regulating qi and eliminating phlegm, and awakening the mind and preventing insanity could be achieved. Take the main acupoints: Renzhong, Yamen, and Dazhui; with auxiliary acupoints to take Baihui, Sishencong, Neiguan, Shenmen, Zhongyuan, Fenglong, Taichong, etc, once a day, 10 times for a course of treatment. The total effective rate was $95.45 \%$. Wei Zheng, et $a l .{ }^{[9]}$ believed that acupuncture could improve the cognitive function of patients with chronic schizophrenia. Seventy patients were selected and divided into acupuncture group and control group. The control group took antipsychotic drugs before entering the group and maintained the effective dose unchanged. At the same time, the acupuncture group combined acupuncture on the basis of the control group and took four magic needles, Neiguan (double), Sanyinjiao (double), Yintang points and other points. Each treatment lasted for 30min, 5 times per week, 10 times for 1 course of treatment, a total of 6 weeks. There were statistically significant differences in WMS memory quotient, DST total net points, error rate and WCST persistent error between the two groups after treatment (all $\mathrm{P}<0.05$ ).

\subsection{Acupoint catgut embedding therapy}

Haifang Zhu, et al. ${ }^{[10]}$ randomly divided 90 schizo- phrenic patients into 2 groups. Risperidone oral liquid was used to treat schizophrenia in the control group, and traditional Chinese medicine wire embedding method was used in the observation group. Buried line selected in the Shanzhong, Zhongwan, Zhongji, Guan Yuan, Baihui, Dazhui, Shenting, Yaoyangguan, Mingmen, Jizhong and so on acupoints. Once buried in 15 days and 3 times as a course of treatment. The control group patients with negative symptoms (SANS) score and brief psychiatric scale (BPRS) scores, Hamilton depression scale (HAMD) score and side effect scale (TESS) score were higher than the patients of the observation group, the difference was statistically significant, that the embedding thread therapy of traditional Chinese medicine in the treatment of negative symptoms of schizophrenia clinical curative effect is good. Yazhi $\mathrm{Lv}^{[11]}$ used "embedding thread and adjusting mind" to treat schizophrenia at Jiaji points of Huatuo, which is located at the 1 st to 7 th thoracic vertebrae, and the 4th and 5th lumbar vertebrae to the 1st sacral vertebra. This method proved to be satisified. Shian Li, et al. ${ }^{[12]}$ selected 52 schizophrenic patients and treated them every 30 days, including Fengchi (double), Dazhui, Jinsuo, Jiu Wei, Shenshu (double), Sanyinjiao (double) and Yongquan (double), once a month for four months as a course, which the total effective rate was $88.5 \%$. It is an excellent method to treat schizophrenia, which cost less time and had no side effects, and the curative effect was definite. Wei Zhang, et al. ${ }^{[13]}$ believed that embedding wires was effective in the treatment of intractable auditory hallucinosis. 70 patients were selected and randomly divided into the embedding group and the control group. The embedding group was treated by embedding wires at Tinggong point and combined with chlorpromazine. The embedding wires at Tinggong point was performed once a month and chlorpromazine hydrochloride tablets were taken twice a day. The control group was treated with chlorpromazine hydrochloride alone. The results showed that the score of brief psychiatric symptom evaluation was lower than the control group $(\mathrm{P}<0.05)$, and with significant effect, the dopamine inhibition ability was greatly improved. In concussion, the treatment effect of the embedding group was better than the control group $(\mathrm{P}<0.05)$.

\section{Conclusion}


To sum up, a large number of clinical practice results show that TCM has achieved satisfactory results in the treatment of schizophrenia, and TCM treatment combined with low-dose antipsychotic medication can achieve satisfactory efficacy, which is superior to western medicine alone, and can reduce the amount of antipsychotic medication and side effects to a certain extent. Moreover, TCM treatment is perfect at starting from the whole, focusing on the main contradictions of the onset, and strictly observing the pathogenesis. Both in compatibility of traditional Chinese medicine and acupuncture point should be treated based on syndrome differentiation to make the treatment more focused, so that the key points of the disease can be grasped without neglecting the overall situation. I believed that the position of TCM in the treatment of schizophrenia will be paid more and more attention.

\section{References}

1. Zhao J. The event-related potential study of abnormal cognition function in schizophrenia [master's thesis]. Xi'an: Air Force Medical University; 2013.

2. $\mathrm{Xu} \mathrm{T,} \mathrm{Su} \mathrm{J.} \mathrm{Textual} \mathrm{research} \mathrm{on} \mathrm{theories} \mathrm{of} \mathrm{Dian} \mathrm{and}$ Kuang in Chinese Medicine. Chinese Journal of Basic Medicine in Traditional Chinese Medicine 2011; 17(1): 29-32.

3. Liu C, Zhu L. Efficacy observation of traditional Chinese medicine decoction combined with aripiprazole in the treatment of chronic schizophrenia. Journal of Clinical Medicine in Practice 2019; 23(17): 119-122.

4. Wu S. Clinical observation on first-episode schizophrenia treated with integrated traditional Chinese and western medicine (in Chinese). Journal of Practical Traditional Chinese Medicine 2014; (9): 840.

5. Huang J. Self-made Ningshen Zhendian decoction for 32 cases of schizophrenia (in Chinese). Fujian Journal of Traditional Chinese Medicine 2001; 32(6): 50.

6. Zhao G. A clinical controlled study of acupuncture and moxibustion combined with low-dose antipsychotics in the treatment of schizophrenia (in Chinese). China Health Care \& Nutrition 2013; (8): 174.

7. Liang W. Observation on therapeutic effect of acupuncture on 29 cases of chronic schizophrenia (in Chinese). China Health Industry 2014; (25): 192 193.

8. $\mathrm{Wu} \mathrm{J}$. Acupuncture treatment of 44 cases of mania (in Chinese). Chinese Acupuncture \& Moxibustion 1994; (5).

9. Zheng W, Zhang L, Huang H, et al. Effect of acupuncture therapy on cognitive function of patients with chronic schizophrenia (in Chinese). Modern Practical Medicine 2020; 32(1): 75-77.

10. Zhu H, Sheng Y, Luo J, et al. Clinical analysis of the effect of Chinese medicine embedding on negative symptoms of schizophrenia. Contemporary Medicine 2019; (22): 132-133.

11. Lv Y. Clinical preaentation on 100 schizophrenic cases with treatment of catgut-embedding method in Back-Shu and Jiaji points. Chinese Acupuncture \& Moxibustion 1988; (5): 12-14.

12. Li S, Peng H, Zhang X, et al. Acupoint catgut implantation for 52 cases of schizophrenia (in Chinese). Shanghai Journal of Acupuncture and Moxibustion 2015; (5): 452-453.

13. Zhang W, Sheng L, Du H, et al. A controlled study of catgut embedding at Tinggong point in treating obstinate auditory hallucinations (in Chinese). Journal of Clinical Psychosomatic Diseases 2015; 21(z2): 107. 